\title{
La crise écologique exige une révolution de l'économie des services
}

Jean Gadrey

\section{(2) OpenEdition}

Journals

Édition électronique

URL : http://journals.openedition.org/developpementdurable/6423

DOI : $10.4000 /$ developpementdurable.6423

ISSN : 1772-9971

\section{Éditeur}

Association DD\&T

\section{Référence électronique}

Jean Gadrey, "La crise écologique exige une révolution de l'économie des services », Développement durable et territoires [En ligne], Points de vue (2003-2010), mis en ligne le 02 septembre 2008, consulté le 10 décembre 2020. URL : http://journals.openedition.org/developpementdurable/6423 ; DOI : https://doi.org/10.4000/developpementdurable.6423

Ce document a été généré automatiquement le 10 décembre 2020.

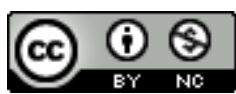

Développement Durable et Territoires est mis à disposition selon les termes de la licence Creative Commons Attribution - Pas d'Utilisation Commerciale 4.0 International. 


\title{
La crise écologique exige une révolution de l'économie des services
}

\author{
Jean Gadrey
}

«La Terre n'est pas un don de nos parents. Ce sont nos enfants qui nous la prêtent »

(maxime amérindienne)

1 L'économie des services, telle qu'elle existe, est, à de rares exceptions près, a-écologique et a-sociale. Elle s'est peu intéressée - sauf dans le cas des transports - aux relations entre les services et l'environnement, et guère plus à la question des inégalités dans et par les services (sauf dans certaines analyses des services publics ou du dualisme de l'emploi tertiaire). Cette situation va se modifier, et probablement de façon rapide, dans les prochaines années. Mais la révolution écologique et sociale de l'économie des services se heurte à divers obstacles, dont certains ne sont pas spécifiques aux services. J'en mentionne quatre, sans chercher à être exhaustif.

2 Obstacle 1 : une prise de conscience freinée, pour de multiples raisons

3 Nous entrons dans une crise écologique et sociale sans précédent historique, pouvant aller jusqu'à un «effondrement» humain mondial. Mais la prise de conscience tarde, parce que les manifestations de cette crise restent limitées, surtout pour les catégories dominantes et dans les pays riches. Mais aussi parce que les solutions, qui existent, heurtent de front les intérêts privés à court terme, la logique elle aussi à courte vue de la mondialisation financière, et celle de la création de besoins superflus par le système de production de l'avidité consumériste, reflété, entre autres, par le montant exorbitant des dépenses de publicité dans le monde, soit près de 500 milliards de dollars prévus en 2008 '.

Obstacle 2: l'économie écologique en est à ses débuts, et elle est peu connue des économistes

5 Il est difficile de penser une économie écologique et sociale (dont celle des services), compte tenu de la nécessité de se projeter bien au-delà du « long terme » des économistes (et même des cycles longs à la Kondratiev) pour intégrer une prospective écologique et humaine sur un siècle au moins, comme c'est le cas, par exemple, du rapport de Nicholas 
Stern (2006). Compte tenu aussi de la nécessité d'intégrer, comme l'a fait cette même étude, les apports de multiples disciplines jusqu'ici ignorées par la plupart des économistes : climatologie, agronomie, sciences de la nature et de la vie en général... à côté des autres sciences sociales.

Obstacle 3 : les services ignorés par l'écologie politique, l'écologie négligée par l'économie des services

L'écologie politique et l'économie de l'environnement, qui disposent d'un riche patrimoine de recherches, s'intéressent peu aux services, à l'exception des transports. Elles ont même parfois considéré les services (en tout cas les services marchands aux ménages) comme des activités serviles. Nombre de leurs concepts clés ont été mis au point en pensant aux biens industriels et agricoles, pas aux services. De son côté, l'économie des services a jusqu'ici pratiquement ignoré les enjeux écologiques. Or on peut montrer qu'on ne résoudra pas la crise écologique sans s'intéresser de près aux services et à leur économie (désormais les trois-quarts de l'emploi dans les pays riches, et presque autant pour la valeur ajoutée), et que l'avenir des services est conditionné par leur rapport à la nature, tout autant que pour les autres activités, mais de façon un peu différente.

Obstacle 4 : remettre les externalités à l'intérieur

Les externalités environnementales deviennent le cœur de l'économie écologique des services, alors que, comme leur nom l'indique, elles étaient à la périphérie de l'économie a-écologique, de fait anti-écologique. Il faudra internaliser les externalités non seulement dans l'évaluation des performances et des coûts, mais aussi en allant jusqu'à les considérer théoriquement comme associées à des biens communs aussi vitaux que les « internalités» que sont les produits et services de type final dont elles dérivent. Quand les dommages collatéraux à terme peuvent devenir plus importants que les bénéfices (en bien-être à court terme) retirés des productions, l'analyse des premiers compte autant que celle des secondes.

Prologue : des constats qui s'opposent

La réflexion sur le rôle des services dans une perspective de durabilité (pour l'instant au sens environnemental du terme) peut débuter en opposant deux types de chiffres, conduisant à des diagnostics radicalement opposés. Pour les premiers, les services seraient des activités étonnamment " vertes ", pour les autres, les économies de services seraient les plus consommatrices de ressources naturelles et les plus polluantes.

Premiers chiffres: en France, les données sur les émissions de CO2, qui font partie des grands marqueurs de pression écologique associés aux risques de changement climatique, sont publiées par secteurs d'activités humaines. On obtient alors les résultats suivants (graphique 1). 
Graphique 1 : Contribution aux émissions de $\mathrm{CO} 2$ par secteur en France

La contribution aux émissions de $\mathrm{CO} 2$ par secteurs, en \%, France, 2005

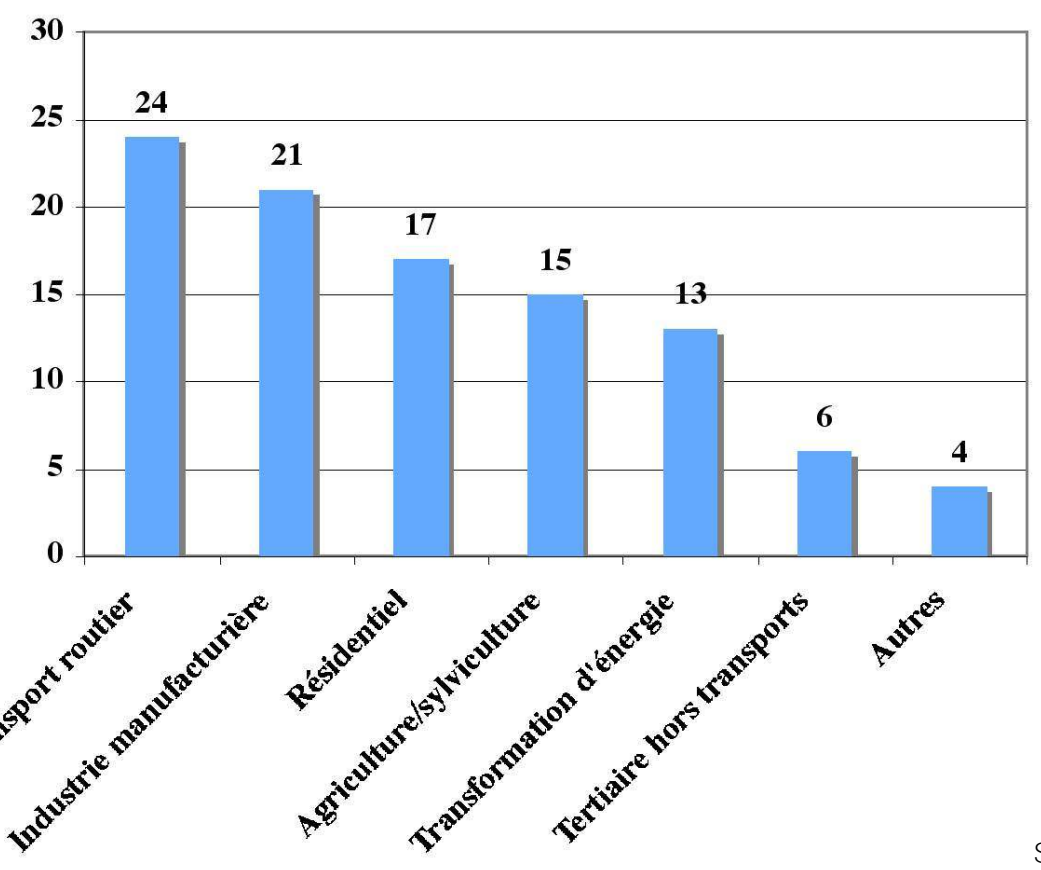

Citepa $2007^{2}$

Source :

Un tel graphique semble donner un avantage déterminant aux services (hors transports) : ils représentent, en 2005, 71,5 \% de l'emploi en France, et ils ne contribueraient que pour $6 \%$ aux émissions, une performance écologique apparemment remarquable au regard de celles des autres secteurs d'activité (le «secteur résidentiel», celui des habitations, n'étant pas un secteur d'activité mais un secteur de consommation). Avec ses $21 \%$ de contribution aux émissions pour un poids dans l'emploi de 13,3\%, l'industrie manufacturière est, selon ces chiffres, 19 fois plus polluante par emploi que les services, l'agriculture (3,6 \% de l'emploi) 50 fois plus, les transports routiers (3,2\% de l'emploi) 90 fois plus, et le secteur de l'énergie (0,8\% de l'emploi) 190 fois plus ! C'est sur la base de tels chiffres que certains ont pu voir dans la "croissance tertiaire » un élément très favorable au « développement durable ».

Seconds chiffres : si les services sont écologiquement très « légers », on pourrait penser que les pays dont l'économie est la plus «tertiaire » se comportent mieux sur le plan de leur pression écologique par habitant. Reprenant la variable des émissions de CO2 par habitant, et la croisant avec le poids des services dans l'emploi, on obtient le graphique 2 suivant, pour 28 pays de l'OCDE et de l'Union européenne (quelques pays manquent à l'appel parce que les données font défaut) : 
Graphique 2 : Emissions de CO2 par habitant (2003-2004) OCDE

28 pays de l'OCDE et de l'UE : part des services dans l'emploi et émissions de $\mathrm{CO} 2$ par habitant en 2003-2004 : une tendance à la progression des émissions avec la tertiarisation

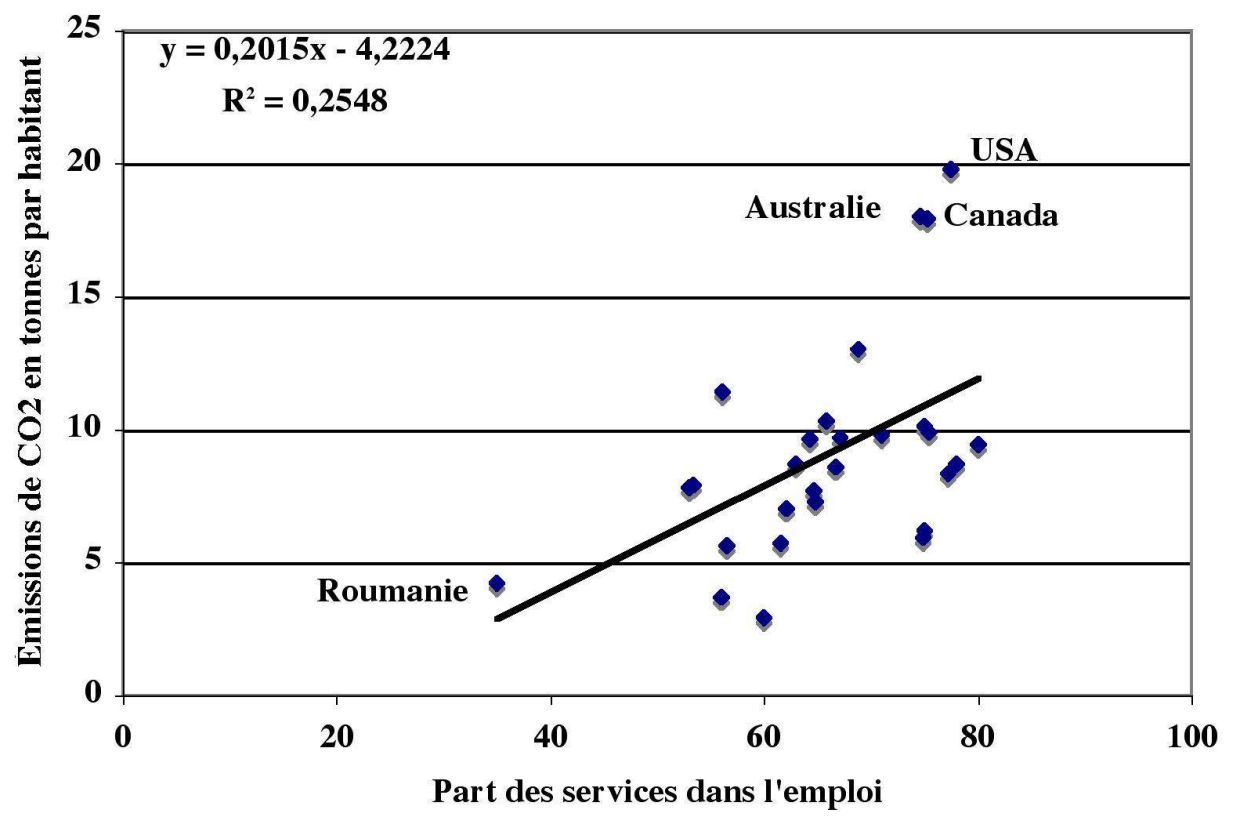

Sources. Emploi dans les services : OCDE et Eurostat ; émissions : PNUD, 2006

Sans être très forte, la corrélation est significative, et la tendance est visiblement à ce que les pays les plus tertiaires soient les plus pollueurs selon ce critère, les records étant détenus par trois pays qui ont refusé de signer le protocole de Kyoto, dont les États-Unis. L'Australie, ravagée par la sécheresse et les incendies, est revenue en 2007 sur ce refus. Même constat, avec une corrélation plus forte, si l'on remplace la variable des émissions de CO2 par l'empreinte écologique par personne (graphique 3) 
Part des services dans l'emploi et empreinte écologique par personne en 2003,28 pays de l'OCDE et de l'UE

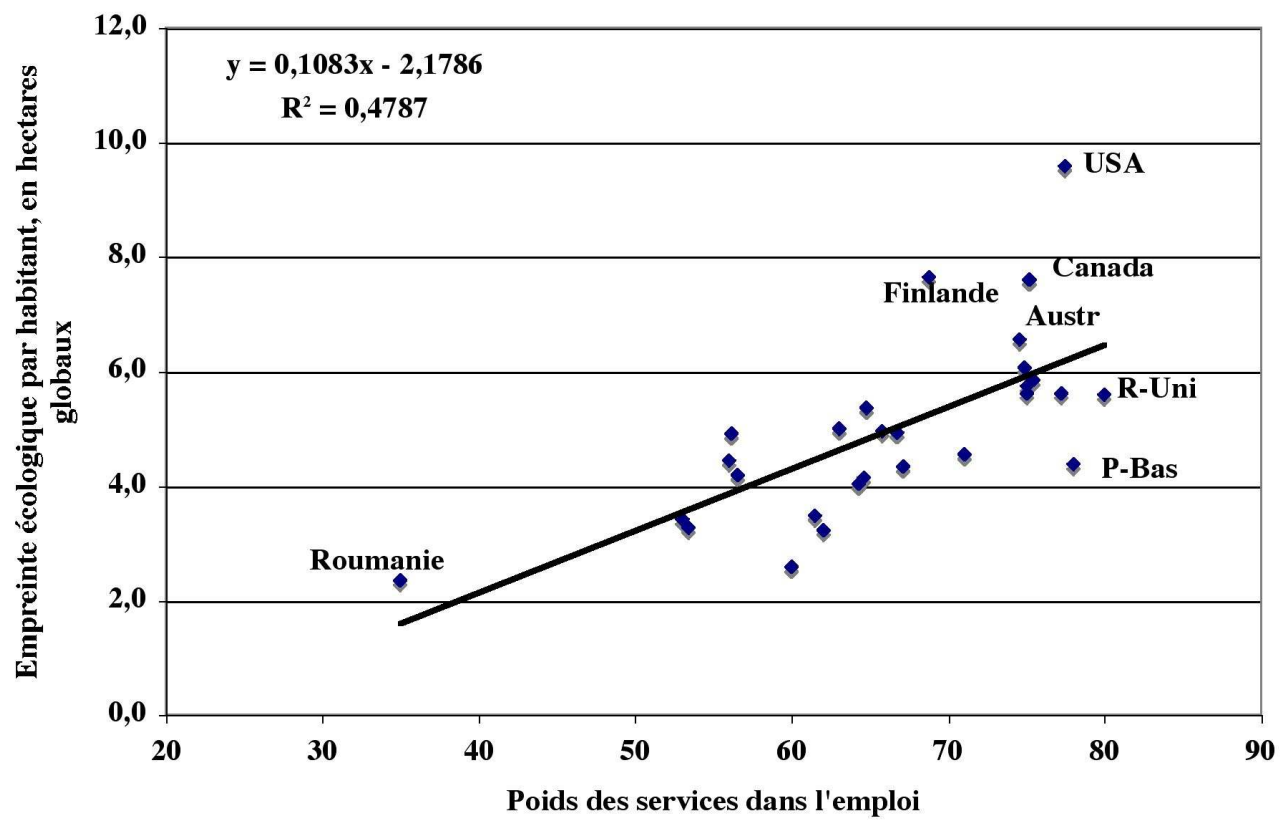

Source : pour l'empreinte écologique en 2003, WWF, rapport « planète vivante 2006 ».

Les constats empiriques précédents, en forme de contradiction, invitent à creuser la question de la pression écologique des services et de sa mesure (section 1), avant de traiter celle de l'avenir de l'emploi tertiaire sous contraintes environnementales (sections 2 et 3 ).

1. La matérialité des services et de leurs externalités, dimension essentielle de leur économie écologique. La dématérialisation est (pour l'instant) un mythe.

L'idée selon laquelle nous assistons à une « dématérialisation de l'économie » qui aurait notamment à voir avec l'expansion des services est une contrevérité. Un service est peutêtre, comme on l'a écrit sur un mode plaisant, « un produit qui ne vous fait aucun mal quand il vous tombe sur les pieds", mais sa production et sa consommation font parfois autant de mal à la planète que celles des autres produits.

17 Si cela est vrai, et nous verrons que tel est le cas, les services ne constituent pas la solution miracle pour la durabilité environnementale de l'économie future. Il est vrai qu'en moyenne leur production consomme moins d'énergie et de ressources naturelles (par emploi par exemple) que celle de l'agriculture, de l'énergie ou de l'industrie. Mais cette moyenne, d'une part, recouvre des situations extrêmement différentes, entre les transports routiers et aériens qui battent tous les records d'émissions de GES (gaz à effet de serre) et des services de proximité relationnels dont la pression écologique est assez faible, sans être négligeable. D'autre part et surtout, elle repose sur un calcul partiel et trompeur qui oublie une caractéristique "matérielle» essentielle de la plupart des services: la présence physique simultanée des prestataires et des usagers qui « coproduisent » le service.

Il ne suffit donc pas d'évaluer la pression écologique des services sous l'angle de leurs facteurs matériels de production au sens strict (bâtiments et chauffage, machines, 
ordinateurs, énergie...), il faut tenir compte des facteurs matériels de la co-production (§ 1.1). Second oubli fâcheux, le principal «facteur de production » des services, le facteur humain, est absent des calculs usuels de la pression écologique des services. Il faut le réintroduire, ici comme ailleurs ( $\$ 1.2$ ). Nous évoquerons ensuite, pour la critiquer, une troisième thèse erronée, celle qui assimile la réduction de la consommation de matières par unité de PIB à une "dématérialisation » de l'économie (1.3). Cette thèse n'est pas spécifique aux services, mais elle les concerne indirectement. Puis nous verrons que, pour l'instant en tout cas, le développement des NTIC, particulièrement important dans les services, ne s'accompagne nullement d'une dématérialisation ou réduction de la pression écologique (1.4).

1.1. La relation de service et son environnement matériel doivent faire l'objet d'une analyse écologique complète

La « relation de service» et la " coproduction » des services ont fait l'objet, à juste titre, de beaucoup d'attention et de travaux de la part des socio-économistes des services. Mais ils ont eu tendance à oublier la matérialité de ces « rencontres ", en privilégiant l'analyse, souvent inspirée de la sociologie interactionniste, des échanges de type verbal, cognitif, contractuel, etc.

Les trois composantes principales de cette matérialité de la relation de service sont les suivantes :

21 1) le déplacement des personnes, indispensable à la fourniture du service : déplacement des prestataires dans certains cas (services à domicile, consultants...), des usagers/clients dans d'autres cas (de loin les plus nombreux : élèves et étudiants, patients, clients du commerce ou des hôtels et restaurants...), et, parfois, déplacement des deux catégories d'acteurs de la relation : accompagnateurs de voyages, taxis et conducteurs de bus... mais aussi déplacements des salariés du tertiaire vers les bureaux des administrations ou les lieux de prestation des organisations de services. Les services ne peuvent pas être rendus sans une multitude de déplacements que l'on a tendance à oublier lorsqu'on se limite à leurs «facteurs de production » classiques. Et la matérialité des déplacements n'est pas seulement celle des flux (émissions des véhicules et des transports collectifs, énergie consommée), c'est aussi celle des stocks des véhicules et des infrastructures, pour la part qui revient à la production et à la consommation des services.

2) la matérialité des espaces de la relation: bureaux, salles d'enseignement, hôpitaux, guichets des banques, etc. Il faut des ressources naturelles pour les construire (le stock), et d'autres (des flux réguliers) pour les entretenir, les chauffer, les éclairer, ....

3) la matérialité des outils techniques d'appui à la relation, au guichet, au comptoir, et plus encore à l'hôpital, ainsi que la matérialité du «back-office ", qui peut être très «lourd», en stock et en flux.

24 La principale explication de l'avantage considérable que détiennent les services dans les données d'émissions de $\mathrm{CO} 2$ par secteur (graphique 1) est que l'on n'y compte que les flux d'émissions associées au chauffage et à l'éclairage des bâtiments, et accessoirement à d'autres utilisations de l'énergie pour les outils techniques. Il ne s'agit que d'une fraction minoritaire de l'usage de ressources matérielles pour produire et consommer les services. Par exemple, dans le bilan carbone effectué en France par l'ADEME pour la chaîne de supermarchés Casino, près de $40 \%$ des émissions de $\mathrm{CO} 2$ tous domaines confondus correspondent aux déplacements des clients. Ce chiffre serait-il très différent pour le bilan carbone d'un campus universitaire ou d'un centre hospitalier, compte tenu de 
l'ampleur des déplacements quotidiens induits par les activités «immatérielles» de l'enseignement, de la recherche, et des soins médicaux ? Serait-il très différent dans le cas des spectacles et des concerts (qui sont des exemples typiques de "services stagnants » selon Baumol, voir la section 2) ? Les chercheurs, si attachés à l'organisation de colloques " de haut niveau ", de préférence internationaux, ont-ils conscience de la contribution de leur production « immatérielle » de savoirs au réchauffement climatique?

1.2. Le bilan écologique des industries de service doit tenir compte de la consommation de ressources naturelles des prestataires

On a tendance à oublier un fait majeur: la croissance des services n'a été possible que parce que, d'un côté, les prestataires de services pouvaient disposer d'un niveau de vie comparable à celui des autres actifs (c'est l'hypothèse de l'égalité des salaires du modèle de Baumol, voir la section 2), pendant que, d'un autre côté, les consommateurs pouvaient dépenser de moins en moins (en proportion de leur budget) en biens matériels de base, et devenir acheteurs de services (c'est ce qu'on appelle parfois la « loi d'Engel»). Cela s'est produit parce que la composante de biens matériels de base de ce niveau de vie (se nourrir, se loger, se vêtir...) a fait l'objet de fantastiques gains de productivité «brute ", lesquels ont été obtenus, surtout au cours des cinquante dernières années, en surexploitant les ressources naturelles et en émettant des déchets et des gaz à effet de serre (GES) au-delà de ce que la nature peut régénérer selon ses propres rythmes. Pour le dire d'une façon très provocatrice, la "société de services", telle en tout cas qu'elle s'est développée, en s'appuyant sur un productivisme « insoutenable » appliqué dans les autres secteurs, mais aussi parfois dans les services, est sans le savoir une société anti-écologique, hyperindustrielle et hypermatérielle.

Ajoutons un autre argument à l'appui de notre thèse de l'hypermatérialité des sociétés de services contemporaines. Si l'on examine uniquement les chiffres d'emploi, on obtient, quelle que soit la conjoncture économique, une progression considérable aussi bien de l'emploi total dans les services que de son poids relatif, au détriment de l'agriculture et de l'industrie. En France, en se limitant à la période des trente dernières années, et en retenant la croissance de l'emploi (indice 100 en 1978), on obtient le graphique 4 suivant : 
Graphique 4 : croissance de l'emploi dans les services, l'industrie et l'agriculture

La croissance de l'emploi dans les services, l'industrie (hors BTP) et l'agriculture, base 100 en 1978

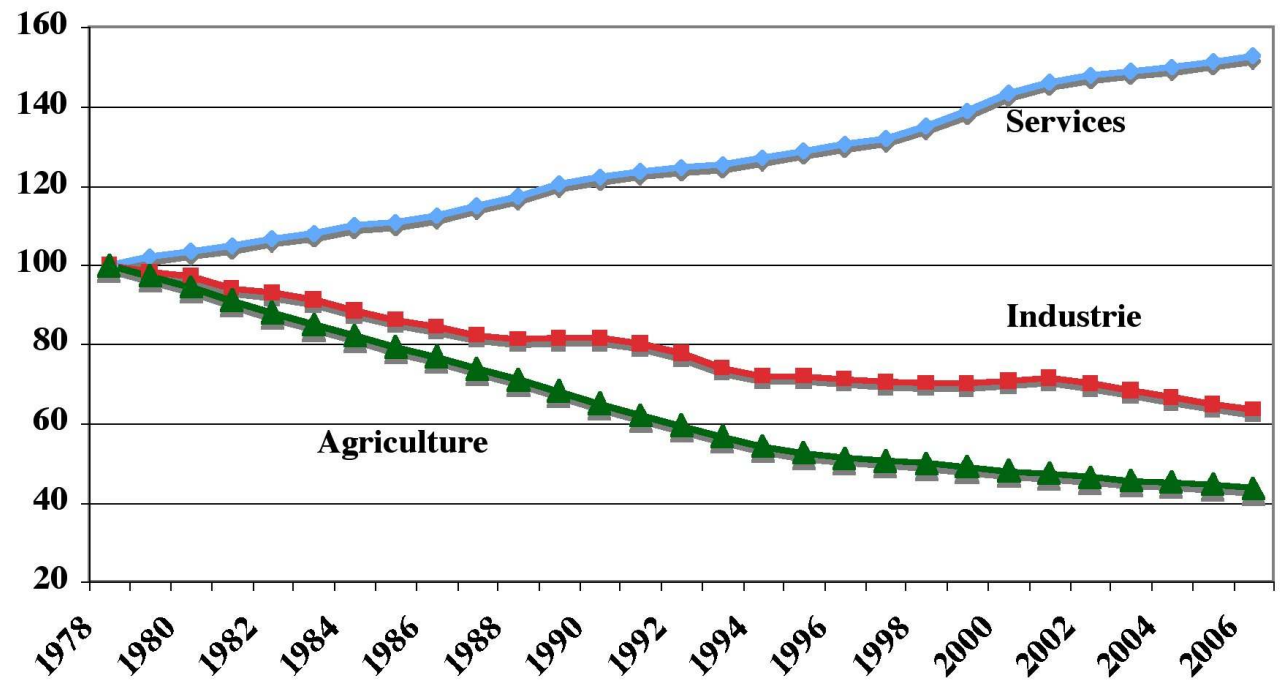

Le tableau est bien différent en ce qui concerne les quantités produites ou consommées (les «volumes", au sens statistique). Si l'on prend par exemple les grands postes de la consommation finale des ménages (hors services publics non payants), on obtient le graphique 5 :

Graphique 5 : consommation finale des ménages (1959)

La consommation finale des ménages en volume, indice 100 en 1959

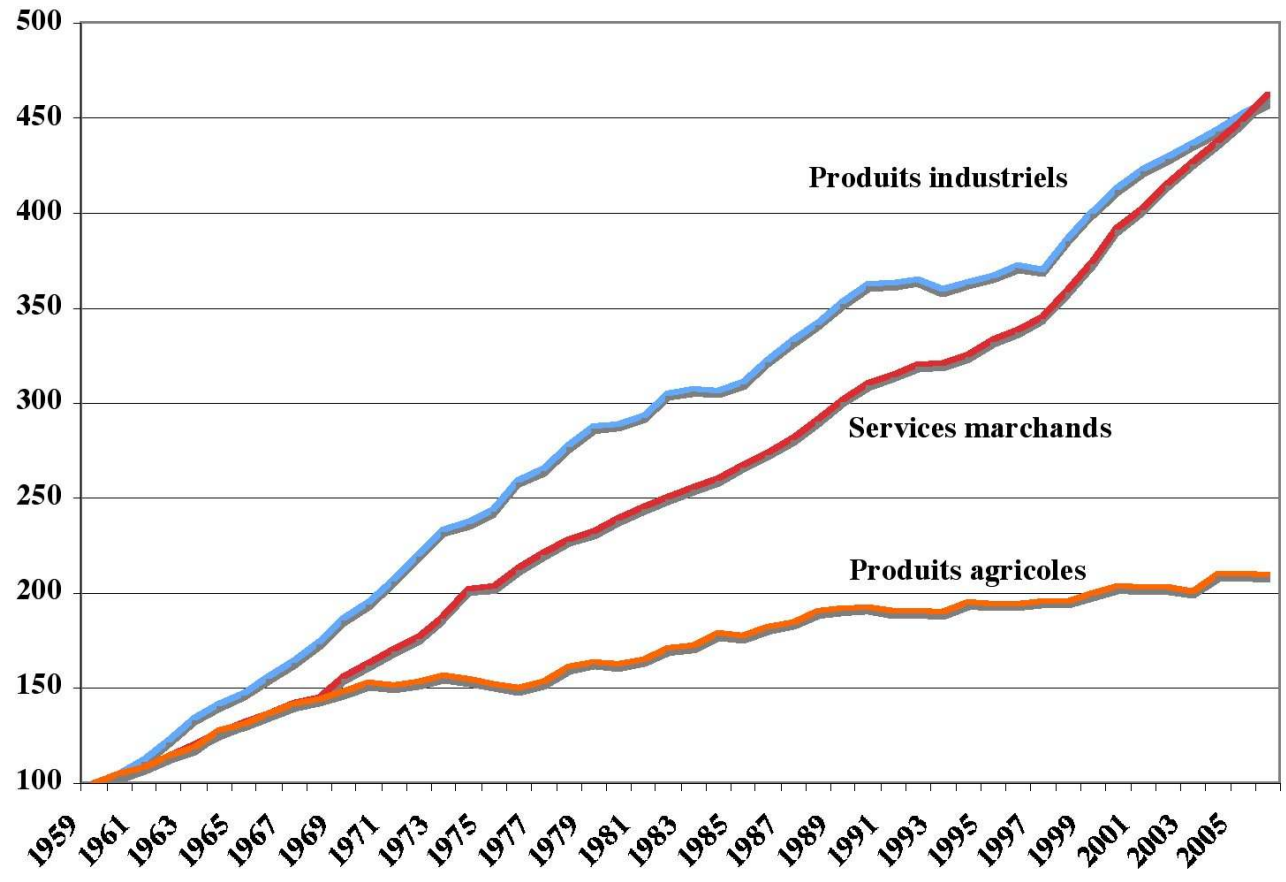


Depuis 1959, le volume de biens industriels consommés par les ménages a été multiplié par plus de 4,5 , tout comme celui des services marchands. Quant aux produits agricoles consommés, la progression de leur volume est certes bien plus faible, mais ce volume a néanmoins plus que doublé. En conséquence, même ceux qui assimileraient la progression de la matérialisation de l'économie à l'importance accrue de la consommation de produits industriels et agricoles devraient considérer qu'un tel graphique supporte l'idée que les "économies de services » (sous l'angle du poids dominant de ces activités dans l'emploi et dans la valeur ajoutée) sont des économies de plus en plus "matérielles». Dans «les économies de services", on produit et on consomme de plus en plus de "biens matériels ».

1.3. La réduction des émissions et des consommations de matières par unité de PIB n'a rien à voir avec la dématérialisation de l'économie

L'idée d'une dématérialisation de l'économie s'appuie parfois sur le constat d'un découplage des évolutions du PIB (en volume) et des indicateurs de pression écologique (utilisation de ressources, émissions et pollutions), ou, ce qui revient au même, sur des chiffres de réduction des volumes de matières et de pollution par unité de PIB. De tels constats ne manquent pas d'intérêt, mais ils passent complètement à côté de la question de la finitude des ressources naturelles renouvelables et de l'épuisement des ressources non renouvelables. La seule chose qui compte pour apprécier si nous vivons dans des sociétés dont le "matérialisme objectif » progresse ou régresse sous contrainte de ressources finies est le volume total absolu des prélèvements et celui des rejets. L'un et l'autre ne cessent de progresser et ont atteint des niveaux bien supérieurs aux capacités de la nature. Les services ne sont pas spécifiquement concernés par cette critique de la thèse de la dématérialisation, mais ils le sont indirectement dès lors que les économies les plus tertiaires sont aussi les plus intensives en usage de ressources naturelles.

Graphique 6

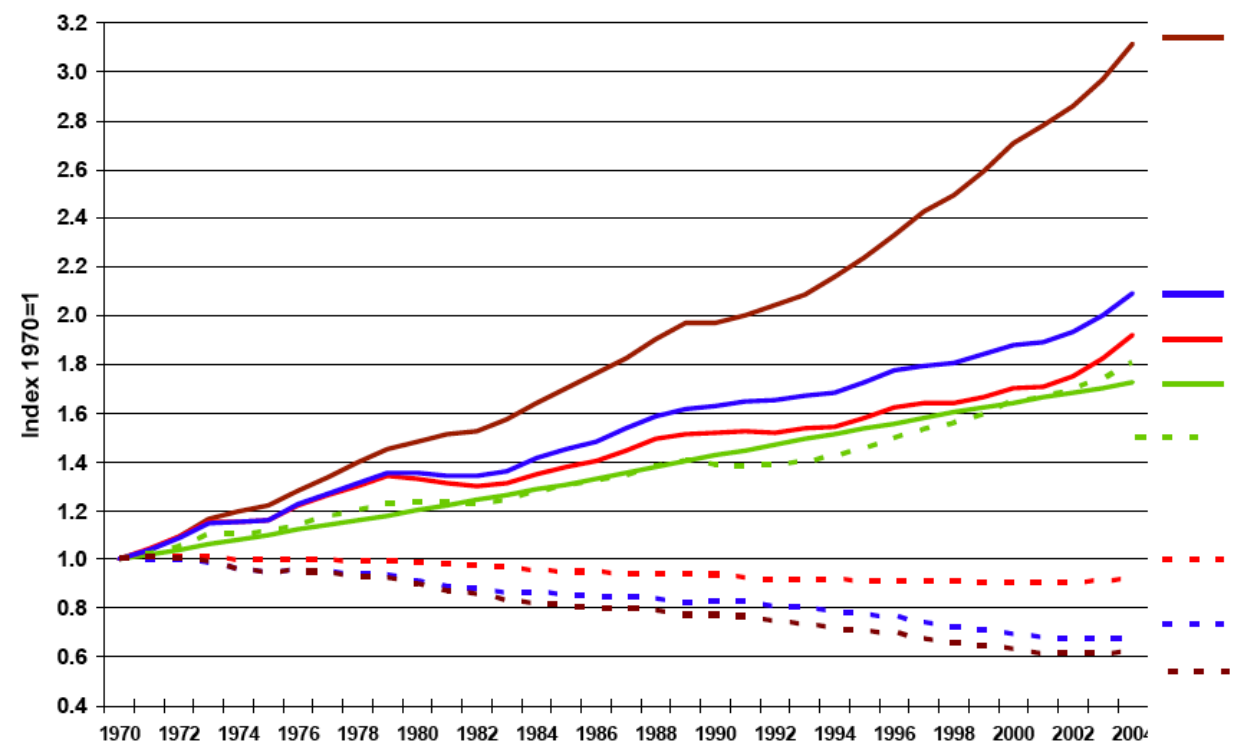

Source : Jean-Marc Jancovici, « La dématérialisation de l'économie : mythe ou réalité ? », La jaune et la rouge, $n^{\circ}$ spécial « Croissance et environnement », août-septembre 2007.

1.4. Les NTIC, réponse à la durabilité des services? 
Les NTIC, utiles à certains égards, ne constituent pas non plus « la » solution pour assurer la durabilité des services. Leur empreinte écologique est et restera lourde, car elles ne sont nullement immatérielles, bien au contraire. Je me limite à deux indices. Le premier porte sur la matérialité de la production des ordinateurs (il y aurait aujourd'hui environ 1,5 milliards d'ordinateurs en fonction dans le monde), le second sur l'évolution comparée des flux d'information et des flux de transports depuis 1800 . présentes dans l'ordinateur comme produit fini, ou même les flux d'énergie nécessaires à son fonctionnement, bien que cela devienne non négligeable (voir ci-après), que les flux requis pour sa production. Les ordres de grandeur disponibles sont les suivants. Les émissions de $\mathrm{CO} 2$ liées à la production et au transport d'un ordinateur de bureau (poste fixe) à écran plat fabriqué aux États-Unis ou en Asie seraient de 1,3 tonnes de CO2, dont la moitié pour son transport en avion. On rappelle que, dans un monde parfaitement égalitaire, avec la population actuelle, le «droit d'émission durable » de chaque habitant serait de 1,8 tonnes de CO2 par personne et par an. C'est ce que la nature peut absorber sans aggravation de l'effet de serre. tonne de matériaux divers pour la production d'un tel ordinateur, dont 240 kilos d'énergie fossile et 1500 litres d'eaus. "L'empreinte eau » d'une simple puce de 32 Mo et de deux grammes (qu'on appelle aussi «l'eau virtuelle» contenue dans cette puce) est d'environ 32 litres ${ }^{6}$. Sous ces divers angles, le «poids écologique » d'un simple ordinateur est élevé, et cela fait réfléchir à un autre aspect de l'impression de dématérialisation que peut fournir l'observation superficielle des économies modernes de services, à savoir la miniaturisation des composants et des outils techniques de nombre de services. En réalité, ce que les études précédentes mettent en évidence, c'est une forme de " découplage » bien peu écologique : plus on miniaturise, plus on réduit le rapport entre le volume des matières "qui restent dans le produit final » et celui des matières qui entrent dans son processus de production. Ce rapport est, en poids, de un à deux pour une voiture et de un à 630 pour une puce électronique (même source).

Si l'on ajoute à ces bilans des matériaux les flux d'énergie nécessaires au fonctionnement de l'informatique et autres technologies de l'information, le tableau s'assombrit encore un peu : «L'empreinte énergétique des technologies de l'information s'impose comme un enjeu majeur. Selon l'Agence Internationale de l'Energie, les équipements électroniques et leurs mises en veille sont devenus l'un des tous premiers postes de consommation électrique des ménages. Dépassant désormais le poste traditionnel « froid », et talonnant, chez les foyers équipés, celui du chauffage électrique. »....

La croissance des flux d'information n'a pas réduit dans le passé la circulation des flux de matières

Dans le passé en tout cas (période 1800-1985), ces deux flux ont connu une expansion parallèle. Leur découplage est possible, et même très probable depuis 20 ans, mais pas au point d'aboutir à une substitution partielle qui verrait une décroissance des transports de biens.' Nous reviendrons par la suite sur le cas des transports et de leur forte progression dans la période récente. On pourrait faire des constats semblables, depuis les années 1980, 
en ce qui concerne le rapport entre l'utilisation de l'informatique et la consommation de papier, ou encore l'expansion préoccupante des déchets informatiques.

2. La tendance à la progression de l'emploi tertiaire sera affectée voire inversée

Cette hypothèse constitue une très sérieuse remise en cause d'une théorie plutôt «productiviste » et «a-écologique » que j'ai défendue, comme tous les spécialistes de l'économie des services, depuis le milieu des années 1980 et jusqu'à une période très récente.

La tendance historique observable (au moins à partir du $19^{\text {ème }}$ siècle) à la progression de la part des services dans l'emploi et la valeur ajoutée sera affectée, et peut-être inversée dans les pays développés, tout comme la tendance à la croissance économique et à celle de la productivité, telles qu'elles sont définies et mesurées aujourd'hui. Cette inversion historique possible, qui serait une révolution, peut être analysée, dans un premier temps, en se demandant ce qu'il adviendrait de la théorie économique qui fait aujourd'hui autorité pour expliquer la croissance de la part des services dans l'emploi si l'on y introduisait les externalités environnementales (§ 2.1). Nous verrons ensuite (§ 2.2 et suivants) qu'il faut aller plus loin, et réviser les bases conceptuelles de ces raisonnements, à commencer par la notion de gains de productivité, très insuffisante dans sa définition actuelle pour une prospective de la durabilité écologique et de la place future des services dans l'emploi.

2.1. La « loi de Baumol » revisitée : les prix relatifs des services « stagnants » ne sont pas condamnés à augmenter sans cesse, si on internalise les externalités

Commençons donc par adopter comme point de départ les approches classiques (depuis Clark, Fisher et Fourastié) de l'écart structurel des gains de productivité entre l'industrie (et l'agriculture "industrialisée ») et la majorité des services. Ces approches ont ensuite pris la forme, en 1965, d'une modélisation simple qui a fait le tour du monde, sous le nom de « loi de Baumol». Or cette loi devrait être revisitée parce que le principal concept sur lequel elle repose, celui de productivité du travail, est dans l'incapacité, en tout cas dans son acception actuelle, de tenir compte des externalités, notamment écologiques. Or, ces dernières devront impérativement être internalisées pour gérer le principal enjeu du 21 ème siècle: la rareté croissante de ressources naturelles dans lesquelles la «société industrielle ", puis la " société de services ", ont puisé et rejeté en les considérant comme gratuites (exemple : les forêts comme « puits de carbone ») ou disponibles à faible coût (le pétrole, l'eau). Voici une démonstration non formalisée de la façon dont le modèle de Baumol est alors affecté.

Le modèle de Baumol repose sur trois hypothèses : 1) les gains de productivité du travail sont en moyenne élevés dans la plupart des activités industrielles et agricoles (le " secteur progressif ») et faibles ou nuls dans les services ou en tout cas une bonne partie d'entre eux (le «secteur stagnant») ; 2) les salaires tendent à s'égaliser dans les deux types de secteurs. De ces deux hypothèses, il résulte que le prix relatif des services du secteur stagnant ne cesse de croître par rapport à celui des biens industriels et agricoles. Une troisième hypothèse plus hardie intervient alors, conforme à certaines observations empiriques' : la demande relative de services ne faiblit pas, en volume, par rapport à celle de biens industriels ou agricoles, en dépit de la progression du prix relatif des services. On en déduit assez simplement que la part de l'emploi dans les services «à productivité stagnante » ne peut que croître, sans autre limite que celle de la validité des hypothèses... 
42 Le raisonnement doit être révisé dès ses deux première étapes si d'importantes externalités environnementales sont internalisées (via des taxes ou autres dispositifs politiques), car alors le coût unitaire des biens du secteur progressif va être majoré en fonction des « matières » et des rejets par unité de produit, indépendamment de l'évolution de la productivité du travail.

43 Si par exemple la productivité du travail continue à progresser rapidement dans l'industrie, la «valeur-travail » (coût de production en travail) contenue dans chaque unité de produit industriel va bien évoluer en proportion inverse des gains de productivité, mais la «valeur écologique » (coût environnemental désormais internalisé) de chaque unité restera identique (hypothèse dont la vraisemblance est examinée par la suite). Tout se passe comme si l'on avait intégré dans la production une part fixe de "service stagnant ", ici une sorte de "service de la nature », laquelle refuse obstinément de faire des gains de productivité dans son «travail » (par exemple absorber de plus en plus de $\mathrm{CO} 2$ par hectare de forêt...), ce qui est en effet la cause de nos soucis. Mais alors, le premier résultat de Baumol doit être revu : le prix relatif des biens industriels par rapport à celui des services stagnants n'est plus condamné à tendre vers zéro. Bien au contraire, il tend asymptotiquement vers une limite non nulle, à cause du coût fixe unitaire écologique de la production industrielle. La conclusion n'est pas modifiée si l'on tient compte du fait que les services stagnants ont eux aussi des coûts écologiques de production.

Cette remise en cause du modèle serait sans objet si l'on pouvait supposer que, dans le secteur progressif (ici l'industrie), les coûts politiques (ou tarifs) des «services de la nature " par unité produite, au lieu d'être plus ou moins fixes, comme on vient de le supposer, tendaient eux aussi vers zéro (par rapport au coût des services stagnants). Cela pourrait se produire si, d'une part, les économies de matières, d'énergie et de rejets (les économies de pression écologique par unité produite) progressaient continûment, et si, d'autre part, le coût des services de la nature (par exemple le coût politiquement fixé de l'absorption d'une tonne de carbone) ne progressait pas, ou s'il progressait moins vite que les économies de ressources naturelles et de pression écologique par unité produite.

Or, la tendance des décennies futures sera certainement à la réalisation d'économies de ressources naturelles et de réduction des rejets par unité produite. En revanche, rareté croissante oblige, et prévention des dommages à terme oblige aussi, on assistera très probablement à l'augmentation progressive du prix relatif (ou «tarif ») des « services de la nature ", qu'il s'agisse de ceux que les entreprises et les administrations paient déjà en achetant (sur des marchés régulés) de l'énergie (dont le pétrole), de l'eau, etc., ou de ceux, politiquement déterminés, et qui vont progresser aussi, qu'elles vont payer (cela existe déjà) sous la forme de l'internalisation des externalités: taxes, contraintes publiques, marchés de permis d'émission, etc. Il semble donc très improbable que les coûts des services de la nature par unité produite dans l'industrie (ou l'agriculture) puissent connaître à l'avenir une décroissance continue. L'hypothèse de leur constance, envisagée au départ, est en réalité « optimiste » pour les décennies de transition.

Ce scénario est d'autant plus probable que, même si, dans l'industrie, on parvient à nettement dématérialiser les produits (au sens de leur contenu en ressources naturelles et de leurs rejets), par exemple par la réutilisation et le recyclage des matériaux, en revanche, dans l'agriculture, la sylviculture et la pêche, cette éventualité est presque exclue, sauf pour les rejets et pollutions. On ne dématérialisera pas une baguette de pain blanc sous l'angle de la quantité de blé ou de farine nécessaire, même si l'on sait que le 
recours à l'agriculture biologique et à la proximité peut diviser par deux ou plus les émissions de GES associées.

l'emploi selon les secteurs. En effet, si par exemple les prix relatifs des biens agricoles refusent de baisser (voire augmentent) sous l'influence soit de taxes écologiques (eau, énergie, transports, biodiversité...) soit de l'insuffisance de l'offre (conflits d'usage des terres, désertification, etc.), ce n'est pas pour autant un facteur conduisant à une progression du poids de l'emploi agricole. Les taxes ne font pas en elles-mêmes progresser la valeur ajoutée hors taxes ni les volumes produits et demandés, décisifs pour l'emploi. Quant à la hausse des prix par insuffisance de l'offre par rapport à la demande, elle n'a pas non plus cette propriété de favoriser l'emploi si l'on reste dans une logique productiviste. Elle peut fort bien conduire à l'apparition de rentes agricoles (comparables à la rente pétrolière actuelle), sans effet positif sur l'emploi.

51 Il faut donc pousser plus loin ce raisonnement, en réfléchissant, sous l'angle de l'offre, à l'hypothèse suivante: de nombreuses transformations nécessaires de la production (de biens ou de services) s'accompagneront d'une réduction de la productivité du travail, telle qu'on la mesure actuellement. D'éventuelles mesures alternatives ne sont pas à exclure, mais elles n'existent pas encore. Et bien que tous les secteurs soient concernés, l'agriculture, l'énergie, le bâtiment et une fraction de l'industrie seront aux premières lignes. Cela viendra renforcer la tendance à ce que leur poids dans l'emploi cesse de régresser, d'autant qu'une partie des besoins correspondants sont fondamentaux, de sorte que la demande restera forte. Ce poids devrait même progresser nettement dans certaines branches de la « production matérielle ».

L'explication résumée est la suivante: les produits durables, "propres » ou "verts » (biologiques, recyclables, de plus longue durée de vie, impliquant moins de transport et d'énergie, etc.) exigent et exigeront plus de travail par unité produite que les produits pollués ou polluants issus des procédés productivistes. Ne plus surexploiter la nature et gérer ses «services" avec précaution est bon pour l'emploi (au moins dans certaines branches où la demande ne devrait pas faiblir) mais mauvais pour la productivité du travail «brute », celle qui n'enregistre pas les gains ou les pertes de qualité écologique des produits. 
$53 \mathrm{Au}$ total, selon les hypothèses que nous allons explorer, et pour des raisons qui vont audelà de celles que nous avons mises en avant en revisitant le modèle de Baumol, les services ne seront plus les seuls grands "gisements d'emplois» du futur, même si certains d'entre eux conserveront cette qualité en réponse à des besoins sociaux de proximité faisant l'objet d'innovations de durabilité. Il faut, pour une prospective de l'emploi durable, cesser de raisonner par grands secteurs (primaire, secondaire, tertiaire) et s'intéresser à des niveaux assez fins de branches et de besoins, en tenant compte de la durabilité environnementale de la production et de la consommation, mais aussi des questions sociales que suscitera cette "grande transformation» vers une économie écologique.

Commençons par développer un exemple majeur. L'emploi devrait croître globalement dans l'un des grands secteurs que tous les raisonnements classiques, dont celui de Baumol, condamnaient à un déclin inexorable sous l'effet des gains de productivité : l'agriculture ${ }^{10}$. Ce détour un peu long par l'agriculture dans une analyse consacrée aux services peut surprendre. Mais, d'une part, le raisonnement que l'on y tient est généralisable à d'autres productions et il fait réfléchir aux impasses des mesures de productivité «brute». D'autre part, il faudra bien que les économistes des services retrouvent « le sens de la terre » et redécouvrent que les prestataires de services, dans le monde, doivent d'abord pouvoir se nourrir dans un contexte de souveraineté alimentaire à reconquérir. Nous allons droit, si rien ne change, vers une crise alimentaire mondiale qui concerne aussi bien le Sud que les pauvres des pays riches. Cela ne serait pas bon pour les services.

2.3 L'agriculture : une révolution verte créatrice d'emplois en grand nombre, pour contrer la crise alimentaire mondiale en respectant l'environnement

Dans le passé, la principale façon de faire croître la quantité de production économique (telle qu'on la mesure actuellement) a été la réalisation de gains de productivité du travail, qui se résument par la formule: produire plus des mêmes choses avec la même quantité de travail. Jean Fourastié avait montré par exemple que s'il fallait autour de 200 heures de travail pour produire un quintal de blé de l'an 1000 jusqu'au $18^{\text {ème }}$ siècle, avec de fortes variations selon les années, il n'en fallait plus que 30 vers 1950. On en est aujourd'hui à 2,5 heures avec les techniques les plus "productives", en comptant le temps de travail nécessaire à la production des machines et des «intrants » de la production $^{\text {". }}$ Remarquable contribution à la croissance! Dans les pays « riches", $2 \%$ à 3 $\%$ d'actifs suffisent à nourrir tous les habitants, et parfois plus si le solde du commerce extérieur est positif ! Oui, mais...

Le raisonnement de Fourastié, repris par d'autres économistes, repose sur une hypothèse centrale : la tonne de blé moderne est « la même chose » que la tonne de blé ancienne. En apparence, tel est le cas, et l'on peut même probablement invoquer certaines propriétés physico-chimiques attestant de la supériorité des méthodes modernes, si l'on s'en tient aux grains de blé ou à la farine.

57 Or le blé moderne est issu d'une agriculture de plus en plus intensive qui produit, en même temps que le blé, de considérables externalités négatives. Elle utilise massivement des intrants chimiques, pesticides et autres, dont on commence à évaluer les impacts négatifs sur la santé, sur la mise à mal des écosystèmes, et sur la disparition d'insectes pollinisateurs pourtant essentiels pour bien d'autres productions. Elle contribue (avec d'autres) à épuiser et polluer les nappes phréatiques et à accentuer la désertification, ce 
qui réduit les deux principales ressources de la production agricole: les terres arables disponibles et l'eau. Elle a remplacé l'énergie humaine par de l'énergie "machinique » à base de pétrole : c'est cette substitution du capital (et du pétrole) au travail qui explique la plus grande partie de ses gains de productivité. Les plus gros tracteurs ou moissonneuses batteuses ont une puissance de 500 ch et des moteurs d'une cylindrée de 10 à 12 litres. Tout cela s'accompagne d'émissions de $\mathrm{CO}_{2}$, et le pétrole est en voie de raréfaction. Enfin, comme la distance s'accroît entre les lieux de la production agricole à grande échelle et les lieux de transformation et de consommation, le blé ou la farine modernes exigent du transport, lui-même gros pollueur.

Donc le blé intensif « incorpore » toute une série de caractéristiques négatives au regard de la durabilité. Les mesures de la productivité et de la croissance les ignorent. Pour en tenir compte, il faudrait déduire de la valeur de ce blé "pollueur» la valeur estimée des nombreux dommages collatéraux que sa production et son transport entraînent, et tenir compte de la « valeur d'option » (valeur attribuée aux usages dans le futur) des ressources non renouvelables utilisées (le pétrole surtout). C'est délicat, mais c'est envisageable. Qu'obtiendrait-on? Difficile à dire en l'absence regrettable de tels travaux, mais, en s'inspirant des différences de prix entre l'agriculture intensive et l'agriculture biologique, on peut grossièrement estimer que la productivité ainsi «corrigée " de l'agriculture intensive serait divisée par deux ou plus par rapport à sa valeur brute, en fonction du mode d'évaluation des dégâts écologiques et de la déplétion du pétrole. Ses "véritables " gains de productivité dans la période récente, celle où elle a le plus malmené la nature, seraient probablement faibles ou nuls. Plus généralement, selon des indicateurs existants de « PIB verts », la " croissance corrigée » aurait été nulle depuis 1970 aux États-Unis, et les fantastiques taux de croissance de l'économie chinoise pourraient être divisés par deux ou plus...

Si l'agriculture industrielle était convertie par étapes en un système durable, on n'en reviendrait pas pour autant au Moyen-âge. Il s'agirait même d'une "modernisation » exigeant des innovations, mais il faudrait envisager un retournement inattendu : l'emploi dans l'agriculture, qui n'a cessé de décroître depuis la révolution industrielle (il ne représente guère plus de $3 \%$ de l'emploi total en France, contre $27 \%$ en 1954), devrait augmenter pour satisfaire une demande durable, avec des "prix durables» (plus élevés) incorporant les exigences nouvelles. Il en irait de même de la valeur ajoutée de ce secteur : son poids dans le PIB progresserait nettement. Cette hypothèse concernant la production repose implicitement sur une seconde hypothèse relative à la demande : les ménages augmenteraient leur «coefficient budgétaire " pour ces produits alimentaires devenus à la fois plus chers et durables, ce qui suppose de réfléchir à une notion de " pouvoir d'achat durable » et de réduire les inégalités de revenu pour que ces produits durables restent accessibles à tous.

On aurait alors une forte baisse de la productivité, mesurée selon les méthodes actuelles, mais l'emploi progresserait, à production donnée en quantités brutes (par exemple les quintaux de céréales). Croissance zéro (dans ce secteur), emploi en hausse ? Cela semble difficile à croire. C'est pourtant possible si l'on tient compte des nécessaires gains de qualité et de durabilité, nouveaux grands gisements d'emplois du «développement durable ». En réalité, si l'on était capable d'intégrer les gains de qualité et de durabilité dans les mesures des variations de prix et de productivité, il est probable que l'on assisterait non pas à une baisse de la productivité, mais plutôt à des gains. 
61 En France, une agriculture durable et de proximité, avec pour objectif un haut degré de souveraineté alimentaire, ce qui ne veut pas dire le protectionnisme intégral, pourrait représenter à terme entre 1,5 et 2 millions d'emplois contre moins d'un million aujourd'hui' ${ }^{12}$. Il n'y a en France que $1,7 \%$ de fermes biologiques contre $9 \%$ en Autriche. Il faudrait passer à 10 ou 20 fois plus. Par ailleurs, l'exploitation raisonnée et durable de la biomasse (l'une des multiples énergies renouvelables, dont fait partie le bois-énergie) pourrait à elle seule exiger 150000 emplois agricoles nouveaux.

2.4. Deuxième exemple : les énergies renouvelables et les activités d'économie d'énergie devraient créer, vague d'innovation à l'appui, des centaines de milliers d'emplois dans l'agriculture, l'industrie et les services.

On commence à disposer de scénarios de bonne qualité concernant la montée en puissance des énergies renouvelables, en France et dans beaucoup d'autres pays. Le scénario 2006 pour la France de l'association négaWatt ${ }^{13}$ (110 experts et praticiens) combine 1) le recours croissant à des technologies de production d'énergie propre (les renouvelables), 2) la recherche d'efficacité énergétique (des bâtiments, des appareils : consommer moins de Kwh pour un même usage, par exemple moins de Kwh pour une température donnée des bâtiments), et, 3) la sobriété énergétique (réduction des gaspillages). Ce n'est pas un scénario de régression, au contraire : entre 2000 et 2050, l'usage de l'électricité doublerait à consommation de Kwh stabilisée (efficacité et sobriété), la mobilité (transports) progresserait de 15 \% (moyennant un doublement de l'usage des bus et des trains). Les émissions de gaz à effet de serre liées à la production et consommation d'énergie seraient divisées par plus de 4 par rapport à 2000 .

Dans ce scénario, l'emploi augmenterait nettement dans le bâtiment (construction et surtout réhabilitation), la fabrication de composants et de matériaux, les études technicoéconomiques, les services énergétiques et la maintenance. Surtout si l'on aide ces branches naissantes par des investissements publics et par une fiscalité incitative du côté de la demande.

Le programme de réhabilitation des logements créerait plus de 100000 emplois à temps plein, l'éolien créerait plus de 200000 emplois d'ici 2050, le photovoltaïque 150000 et l'exploitation raisonnée de la biomasse permettrait de maintenir en activité 150000 agriculteurs. Ces emplois, pour la plupart non délocalisables, contribueraient au développement local. Cela fait autour de 600000 emplois ajoutés.

Mais qu'en est-il, dans ce scénario, des gains de productivité et de la croissance, tels qu'ils sont actuellement mesurés ? La croissance de l'emploi et la stagnation du nombre de Kwh produits conduiront à enregistrer une nette baisse de la productivité et une croissance zéro du volume de la production, comme dans le scénario précédent pour l'agriculture. Mais de tels calculs passent à côté de deux facteurs essentiels. Le premier est qu'avec un Kwh, on peut obtenir deux fois plus d'usages (ou de "service final»), or ce sont ces derniers et eux seuls qui comptent dans le « bien-être matériel » lié à l'énergie. Le second est qu'un Kwh "propre» n'est pas un Kwh «sale»: le premier ne produit pas les externalités négatives du second. Pour pouvoir refléter ce qui compte vraiment en termes de bien-être durable (la valeur d'usage durable), il faudrait que la mesure des gains de productivité (et donc des prix unitaires, du pouvoir d'achat, etc.) soit capable d'enregistrer le "volume» de l'utilité finale et les gains de durabilité écologique (par exemple les coûts des dommages évités à long terme). C'est une piste à creuser. Il existe des indices hédoniques, sans doute très imparfaits, qui permettent de tenir compte du 
fait, par exemple, qu'avec un ordinateur actuel on « en a plus » (d'usages, de fonctions, de puissance, de rapidité, etc.) qu'avec un ordinateur d'il y a dix ans. On pourrait tenter de subvertir cette méthode, moyennant des conventions, pour qu'il soit possible de rendre commensurables un Kwh propre et un Kwh sale, et bien d'autres produits et services à "verdir » et à mieux utiliser : productions agricoles polluantes contre productions peu polluantes, kilomètres x passagers propres ou polluants dans les transports, etc.

Comme le cas de l'énergie et de l'agriculture ne sont pas isolés, on peut faire l'hypothèse que parmi les principaux gisements d'emplois utiles de l'avenir, on trouvera, dans tous les secteurs les gains de qualité, d'efficacité d'usage, et de durabilité écologique (diminution des externalités négatives). Dans de tels cas, les mesures actuelles des gains de productivité et de la croissance offriront une image biaisée du "progrès", voire afficheront une décroissance qui n'en serait pas une si l'on pouvait tenir compte de toutes les composantes bien réelles et évaluables d'une «valeur ajoutée d'usage durable » des biens et des services.

\subsection{La proximité comme gisement d'emplois durables}

Il est désormais évident pour tous ceux qui s'inquiètent de l'état actuel et futur des ressources naturelles qu'il faut privilégier autant que possible des activités économiques de proximité, économes en transports, plus riches en liens sociaux, rapprochant non seulement physiquement la production de la consommation, mais aussi les réseaux de producteurs, de distributeurs et de consommateurs, en y faisant fonctionner de la coopération territorialisée. C'est ce que certains appellent la "relocalisation», et elle concerne aussi bien l'agriculture que l'industrie, le bâtiment, l'énergie, et la plupart des services. Nous verrons que les services peuvent jouer à cet égard un rôle de toute première importance : à l'opposé des approches qui ont fait de l'internationalisation des services un moteur de leur expansion, c'est leur relocalisation et la proximité qui seront sans doute leurs principaux atouts.

3. Retour à l'emploi tertiaire : services gagnants, services perdants

Que viennent faire les réflexions de la section précédente dans une analyse prospective des services? D'abord, de façon élémentaire, si certains grands secteurs qui ont connu un déclin continu dans le passé doivent retrouver un poids bien plus élevé dans l'emploi, cela laisse mathématiquement moins d'espace pour l'emploi tertiaire. Argument toutefois insuffisant, car dans les secteurs primaire et secondaire, on trouve certes des branches appelées à se développer, mais, pour d'autres, le déclin est pratiquement assuré à terme. Dans le secteur primaire, la pêche déclinera (raréfaction simultanée des ressources halieutiques et du pétrole), mais aussi, entre autres, l'élevage de bovins. Déclin aussi, par exemple, pour toutes les activités (industrielles ou de service) liées à la «société de l'automobile, du camion et de l'avion » et aux infrastructures correspondantes.

L'avenir de l'emploi dans les services est donc conditionné par 1) la durabilité des activités des secteurs primaires et secondaires, réorientées sur une base non productiviste, 2) des innovations de grande ampleur dans les services eux-mêmes visant à réduire leur empreinte écologique et à développer des services de proximité d'utilité sociale et écologique, et, 3) une décroissance de toutes les productions et consommations dévoreuses d'énergie et d'espace écologique par unité de service final et fortement émettrices de rejets, dont les GES. C'est ce qu'il faut examiner en se situant au niveau des branches, secteurs et filières, en distinguant les «services gagnants » et les «services perdants ».

\subsection{Les services gagnants}


70 Nous avons vu que la proximité, à la fois technique et sociale, allait devenir une composante essentielle de l'organisation sociale de la production et de la consommation durables. Il semblerait donc que les services (hors transports) détiennent sur ce point un avantage comparatif considérable, puisque la plupart d'entre eux sont fournis dans le cadre de « relations de service » impliquant la coprésence des prestataires et des usagers. Mais nous avons également pu critiquer sur un plan écologique cette vision un peu idyllique, qui a tendance à oublier, entre autres, les déplacements induits, mais aussi la matérialité de la « force de travail » des prestataires et de leur empreinte écologique.

Il reste qu'entre la pression écologique des "services de proximité » et celle de la production de biens industriels et agricoles dont les composants parcourent des milliers de kilomètres avant de parvenir sur leurs lieux de mise à disposition et d'usage, il existe en moyenne une nette différence écologique, même si l'on manque de chiffres précis, en particulier sur l'ampleur des déplacements liés aux services. En réalité, les services territorialisés sont très divers, et les transports et externalités négatives qu'ils génèrent dépendent de choix d'organisation et de localisation. La grande distribution n'a pas la même empreinte écologique que le petit commerce de quartier organisé en coopératives liées à des coopératives de producteurs proches. Les services publics qui, sous des "impératifs de productivité », suppriment des bureaux ou agences de proximité pour concentrer leur production dans des centres regroupés, augmentent leurs atteintes à l'environnement tout en diminuant leur utilité sociale. Les services à domicile aux personnes âgées induisent bien plus de déplacements que les lieux de vie ou maisons de retraites de quartier, etc. L'organisation spatiale et sociale de tels services est donc plus importante que leur qualificatif commun de «services de proximité » pour un bilan écologique.

Mais l'avenir de l'emploi dans les services dépend aussi, très largement, d'une évaluation des besoins à venir, en portant un jugement sur l'utilité sociale et écologique de ces besoins, sur leur universalité, et sur leurs modes alternatifs de satisfaction. Il ne fait aucun doute, par exemple, que des besoins légitimes immenses existent en direction des personnes âgées et de la petite enfance, même si les réponses (par exemple à domicile ou non) n'ont pas la même valeur sociétale durable, et dépendent des souhaits des personnes elles-mêmes et de la qualité des options, qualité humaine comprise. Il en va de même dans nombre de services publics. Pour avoir, en France, la densité $e^{14}$ suédoise de « services sociaux » (éducation, santé, action sociale, services aux personnes âgées et à l'enfance, collectivités publiques), il faudrait trois millions d'emplois supplémentaires (soit bien plus que le nombre de chômeurs), et de bien meilleure qualité! Ce chiffre ne signifie pas que l'on peut copier des modèles nationaux, ni que les services suédois en question soient tous écologiquement viables, mais il indique une marge de création d'emplois utiles dont l'écologie devrait être étudiée.

73 Se pose ici la question de l'égalité d'accès à ceux des services, très nombreux, que l'on peut associer à des droits universels existants ou à conquérir, sous contraintes écologiques (un autre droit). Si la densité des « services sociaux » est plus forte dans les pays nordiques, c'est aussi parce l'on y considère que tous doivent pouvoir y accéder, quitte à ce qu'ils soient gratuits ou très peu chers pour les personnes à bas revenus. L'avenir de l'emploi dans de tels services dépend donc aussi, toujours sous contrainte écologique, de choix entre les solutions marchandes concurrentielles, où l'accès est conditionné par les revenus, et les solutions « universelles », qu'elles soient directement 
prises en charge par l'État ou les collectivités locales, ou qu'elles prennent la forme de partenariats avec des associations ou des entreprises.

On estime par exemple qu'un grand service public de la petite enfance exigerait de créer en France un million de places dans les 15 ans à venir et d'ajouter ainsi 150000 emplois. Si l'on ajoute les besoins, nettement plus importants, relatifs aux personnes âgées (aide à domicile, mais aussi et peut-être surtout lieux de vie conviviaux bien insérés dans la cité, les quartiers et territoires), on ne dispose pas d'estimations de ce que produirait l'affirmation d'un droit universel, mais il est certain que cela se chiffrerait en centaines de milliers d'emplois de proximité. Les projections de la DARES par familles de métiers sont de 240000 emplois ajoutés en dix ans. Or elles ne se situent pas dans une optique de services universels de la petite enfance et des personnes âgées. On pourrait alors certainement multiplier ce chiffre par deux ou trois.

D'autres services publics ou universels de proximité, associés à des droits à affirmer, devraient être considérés, notamment pour le logement (avec des emplois à la clé dans les trois grands secteurs), moyennant des bilans écologiques en stock et en flux. Plus généralement, les services publics ont contribué, non sans défauts, à nourrir les territoires. Leur affaiblissement et la disparition de services publics de proximité, outre leur impact écologique négatif, dévitalisent les régions rurales (régions dont les stratégies de durabilité auront besoin) et les périphéries urbaines en crise sociale, renforçant le double mouvement de polarisation des activités dans certaines zones et de désertification pour les autres.

76 Enfin, dans un contexte peu favorable sur le plan des financements publics, l'emploi dans l'économie sociale et associative, qui est essentiellement une économie de services (recoupant partiellement celle des services de proximité aux personnes), a progressé de $15 \%$ depuis 2000 et représente $10 \%$ de l'emploi total, plus très loin de l'industrie avec ses $13,8 \%$. La qualité de l'emploi y est inégale et souvent faible, leur écologie est peu prise en compte, mais leur utilité sociale fait peu de doute dans la plupart des cas.

\subsection{Les services perdants}

77 En tête des services perdants, on trouve les transports routiers et aériens (et, de façon désormais non négligeable, le transport maritime, dont les émissions ont été récemment réévaluées $\left.{ }^{15}\right)$, et avec eux nombre d'autres services et activités agricoles et industrielles qui en dépendent fortement. Il n'est pas besoin d'expliciter les causes de ce déclin annoncé, entre l'envolée du prix des carburants, les taxes à venir sur l'énergie, et l'absence d'alternatives technologiques dans des délais prévisibles et à un niveau suffisant de réduction des GES. D'importantes incertitudes existent, mais le compte n'y sera pas, et d'ailleurs même l'agence européenne de l'environnement (EEA en anglais) vient de l'admettre ${ }^{16}$, en reconnaissant 1) que les explications de l'incapacité de réduire les dommages écologiques de ces transports tenaient à l'organisation, en amont, de secteurs inducteurs de transport (dont les services, mais aussi l'organisation urbaine) et de choix politiques les concernant, et 2) qu'il faudrait envisager leur décroissance. Ce rapport note que de 1995 à 2004, le trafic aérien de passagers a augmenté de 49 \% et celui par voitures individuelles de $18 \%$, la part des déplacements de personnes en voiture s'étant élevée à $74 \%$ en 2004 dans l'Union européenne.

78 Parmi les services les plus dépendants des transports, le tourisme international et le tourisme d'affaires fondés sur le transport aérien vont sombrer, entraînant dans leur chute certaines économies qui en ont fait le pivot de leur développement, sauf si des 
reconversions sont organisées rapidement. Mais d'autres secteur seront touchés, dont le courrier, certaines formules de distribution à forte empreinte carbone, etc. L'organisation internationale de nombreuses activités (dont la recherche...) sera également affectée par ces contraintes. En revanche, un meilleur avenir est promis aux transports les plus doux, y compris le transport ferroviaire des personnes et des marchandises.

3.3 Un tableau de synthèse sur les perspectives de l'emploi à long terme

Le tableau qui suit résume nos hypothèses sur l'avenir de l'emploi tertiaire dans les pays développés au cours des prochaines décennies, dans une perspective de durabilité et d'équité. Il ne reprend pas exactement les classifications usuelles. Les activités des secteurs primaires et industriels sont évoquées mais non développées. Certaines catégories de la colonne de gauche se recoupent. Ce tableau n'autorise pas, en l'état, un bilan prospectif global de l'emploi dans les services, même s'il semble qu'entre les services gagnants et perdants, et compte tenu des deux autres secteurs, il ne faille pas s'attendre à ce que le poids des services progresse à long terme.

\begin{tabular}{|c|c|c|}
\hline Secteurs & Enjeux & $\begin{array}{l}\text { Tendances de l'emploi } \\
\text { d'ici } 2050\end{array}$ \\
\hline $\begin{array}{l}\text { Agriculture, } \\
\text { sylviculture, pêche }\end{array}$ & $\begin{array}{l}\text { Agriculture bio de proximité comme axe } \\
\text { majeur de développement. } \\
\text { Exploitation durable des forêts et de la } \\
\text { biomasse. } \\
\text { Pêche durable. }\end{array}$ & $\begin{array}{l}\text { Croissance globale dans } \\
\text { l'agriculture et la } \\
\text { sylviculture, avec des } \\
\text { contrastes selon les bilans } \\
\text { écologiques des } \\
\text { productions. } \\
\text { Forte décroissance de la } \\
\text { pêche. }\end{array}$ \\
\hline $\begin{array}{l}\text { Industrie } \\
\text { manufacturière }\end{array}$ & $\begin{array}{l}\text { Réduction forte des volumes de matières et } \\
\text { d'émissions par unité de service final. } \\
\text { Relocalisation partielle, prime aux PME de } \\
\text { proximité. } \\
\text { Croissance de nouveaux secteurs fondée } \\
\text { sur des innovations de durabilité des } \\
\text { produits. }\end{array}$ & $\begin{array}{l}\text { Réduction de l'emploi } \\
\text { freinée par une limitation } \\
\text { du productivisme et de la } \\
\text { logique des firmes } \\
\text { globales. Reconversions } \\
\text { possibles dans certains cas. }\end{array}$ \\
\hline Énergie & $\begin{array}{l}\text { Passage volontariste aux énergies } \\
\text { renouvelables. Tarification sociale en } \\
\text { fonction des revenus. } \\
\text { Compromis entre des énergies } \\
\text { renouvelables à forte empreinte spatiale } \\
\text { (biomasse...) et d'autres à plus grande } \\
\text { intensité de matières (photovoltaïque, } \\
\text { barrages, éolien). Passage à une société } \\
\text { sans pétrole. Sobriété et efficacité } \\
\text { énergétiques. }\end{array}$ & $\begin{array}{l}\text { Croissance forte d'ici } 2050 \\
\text { dans ce secteur et dans } \\
\text { tous ceux qui concourent } \\
\text { aux économies d'énergie, } \\
\text { dont le bâtiment, le } \\
\text { chauffage, etc. }\end{array}$ \\
\hline
\end{tabular}




\begin{tabular}{|c|c|c|}
\hline $\begin{array}{l}\text { Bâtiment } \\
\text { urbanisme }\end{array}$ & $\begin{array}{l}\text { Économies d'énergie et de matières, } \\
\text { urbanisme durable, réhabilitation, "villes } \\
\text { lentes », proximité. }\end{array}$ & $\begin{array}{l}\text { Croissance pour les } \\
\text { décennies de transition } \\
\text { (réhabilitation d'abord) }\end{array}$ \\
\hline Transports & $\begin{array}{l}\text { Décroissance organisée des transports } \\
\text { émetteurs de GES et d'autres pollutions et } \\
\text { des productions associées à la société de } \\
\text { l'automobile, du camion et de l'avion. La } \\
\text { vitesse en question. } \\
\text { Montée en puissance des transports plus } \\
\text { doux et plus lents. Réflexions sur les } \\
\text { inducteurs de transport. }\end{array}$ & $\begin{array}{l}\text { Forte décroissance de } \\
\text { l'emploi, sauf dans les } \\
\text { transports collectifs les } \\
\text { plus écologiques prenant } \\
\text { en partie le relais. Au total, } \\
\text { la mobilité pourrait ne pas } \\
\text { décroître. }\end{array}$ \\
\hline Commerce & $\begin{array}{l}\text { Priorité à un commerce de proximité lui- } \\
\text { même lié à des coopérations de } \\
\text { producteurs de proximité. Centrales de } \\
\text { coopération. } \\
\text { Décroissance du système des grandes } \\
\text { surfaces et de leurs horaires asociaux. }\end{array}$ & $\begin{array}{l}\text { Probable croissance de } \\
\text { l'emploi au total, pour une } \\
\text { distribution moins } \\
\text { productiviste et moins } \\
\text { concentrée. }\end{array}$ \\
\hline $\begin{array}{l}\text { Réparation, } \\
\text { recyclage, } \\
\text { entretien... }\end{array}$ & $\begin{array}{l}\text { Parmi les activités les plus importantes de } \\
\text { la dura-bilité. Une partie pourrait } \\
\text { contribuer aux recon-versions industrielles } \\
\text { (industrie «servicielle»). Recyclage des } \\
\text { déchets solides contre incinération, etc. }\end{array}$ & Forte croissance \\
\hline Location de biens & $\begin{array}{l}\text { Permettre un partage écologiquement } \\
\text { efficace de l'usage des biens. }\end{array}$ & Croissance \\
\hline Artisanat & $\begin{array}{l}\text { Rôle important dans la proximité : } \\
\text { réparation, entretien, recyclage... }\end{array}$ & Croissance \\
\hline Postes & $\begin{array}{l}\text { Comment réduire 1) les émissions par unité } \\
\text { de courrier ou de colis, 2) les flux? } \\
\text { Incidences sur la qualité de service, sur la } \\
\text { VPC, etc.. Prime au courrier électronique. }\end{array}$ & $\begin{array}{l}\text { Réduction nette (déjà } \\
\text { entamée, mais sur une } \\
\text { base productiviste aux } \\
\text { multiples externalités } \\
\text { négatives). }\end{array}$ \\
\hline $\begin{array}{l}\text { Télécommuni- } \\
\text { cations et réseaux } \\
\text { informatique, } \\
\text { ordinateurs }\end{array}$ & $\begin{array}{l}\text { Empreinte écologique (matériaux, } \\
\text { émissions) et empreinte eau des terminaux } \\
\text { et des réseaux, valeur d'usage durable. } \\
\text { Recherches sur les dommages sur la santé. } \\
\text { Innovations pour verdir les produits et } \\
\text { infrastructures. }\end{array}$ & $\begin{array}{l}\text { Ralentissement de la } \\
\text { croissance dans les pays } \\
\text { riches, progression } \\
\text { ailleurs, sous } \\
\text { écolontraintes } \\
\text { écolques. }\end{array}$ \\
\hline
\end{tabular}




\begin{tabular}{|c|c|c|}
\hline $\begin{array}{l}\text { Banques, } \\
\text { assurances, } \\
\text { immobilier }\end{array}$ & $\begin{array}{l}\text { Les crises financières et immobilières } \\
\text { pousseront à des formes de contrôle public } \\
\text { ou de propriété publique, tout comme la } \\
\text { nécessité de retrouver une maîtrise du } \\
\text { foncier. Établissements de proximité. } \\
\text { Nouvelles monnaies locales. }\end{array}$ & Réduction \\
\hline $\begin{array}{l}\text { Services aux } \\
\text { entreprises et adm. } \\
\text { hors postes et } \\
\text { télécom. }\end{array}$ & $\begin{array}{l}\text { Pour les «services opérationnels " } \\
\text { (nettoyage, sécu-rité, restauration...) } \\
\text { enjeux écologiques et sociaux. } \\
\text { Pour les prestations intellectuelles, } \\
\text { réduction des déplacements de personnes, } \\
\text { prime à la proximité, nouveaux conseils en } \\
\text { DD, éco-conception... }\end{array}$ & $\begin{array}{l}\text { Croissance lente de } \\
\text { proximité, décroissance de } \\
\text { la publicité et du } \\
\text { marketing. }\end{array}$ \\
\hline $\begin{array}{l}\text { Hôtellerie- } \\
\text { restauration, } \\
\text { tourisme }\end{array}$ & $\begin{array}{l}\text { Secteur frappé par la crise des transports } \\
\text { motorisés sur longue distance, mais prime } \\
\text { au tourisme et à la restauration de } \\
\text { proximité sous contrainte écologique } \\
\text { d'approvisionnement }\end{array}$ & $\begin{array}{l}\text { Réduction probable de } \\
\text { l'emploi, très forte pour le } \\
\text { tourisme « au loin ». Prime } \\
\text { à la restauration de } \\
\text { proximité liée à des } \\
\text { fournisseurs de proximité. }\end{array}$ \\
\hline $\begin{array}{l}\text { Services à la petite } \\
\text { enfance et aux } \\
\text { personnes âgées }\end{array}$ & $\begin{array}{l}\text { Passer à des solutions universelles } \\
\text { associées à des droits sous contraintes de } \\
\text { respect de l'environnement, réflexions sur } \\
\text { les avantages et inconvénients des } \\
\text { solutions au domicile ou en dehors. } \\
\text { Professionnalisation. }\end{array}$ & $\begin{array}{l}\text { Forte croissance } \\
\text { mobilisant des } \\
\text { financements publics. }\end{array}$ \\
\hline $\begin{array}{l}\text { Administrations } \\
\text { locales }\end{array}$ & $\begin{array}{l}\text { Seront de plus en plus cruciales pour la } \\
\text { durabilité. Retour en régie de nombreux } \\
\text { services sous-traités au secteur privé dans } \\
\text { des conditions insatisfaisantes sur le plan } \\
\text { social et écologique. }\end{array}$ & Croissance \\
\hline $\begin{array}{l}\text { Administrations } \\
\text { nationales }\end{array}$ & $\begin{array}{l}\text { Retour à des agences et établissements de } \\
\text { proximité, décentralisation mais avec des } \\
\text { normes de qualité sociale et écologique. } \\
\text { Pour la santé et l'éducation, débat sur les } \\
\text { excès de la course aux diplômes et sur } \\
\text { l'hypermédicalisation, mais exigences } \\
\text { d'égalité fondée sur des droits. De même } \\
\text { pour la justice. }\end{array}$ & $\begin{array}{l}\text { Le partage national/local } \\
\text { évoluera, mais la part des } \\
\text { administrations et services } \\
\text { publics devrait progresser. } \\
\text { Faible progression dans } \\
\text { l'éducation et dans la } \\
\text { santé. }\end{array}$ \\
\hline
\end{tabular}

Conclusion : la « question sociale » du $21^{\text {ème }}$ siècle et les services

La plus importante «question sociale » du $21^{\text {ème }}$ siècle sera celle des incidences sociales mondiales de la crise écologique. C'est ce que montre le rapport 2007-2008 du Programme des Nations Unies pour le Développement (décembre 2008) sur le seul plan du climat 
(encadré). Mais un important rapport antérieur (2005 Millenium Ecosystem Assessment ${ }^{17}$ ) avait traité du cas des incidences humaines de la destruction des écosystèmes.

Le rapport 2007-2008 du PNUD

Dès aujourd'hui, le réchauffement climatique devient l'un des principaux facteurs de "l'arrêt du développement humain", par ses effets sur l'agriculture et sur la sécurité alimentaire (sécheresse), sur les pénuries d'eau, la disparition d'écosystèmes vitaux, l'exposition aux inondations des zones côtières, les risques sanitaires accrus (paludisme, etc.), et sur le renforcement des inégalités sociales. Ce qui est déjà observable va s'aggraver. "Le changement climatique pourrait exposer 600 millions d'individus de plus au problème de la malnutrition d'ici 2080... provoquer le déplacement de 330 millions de personnes du fait des inondations... faire passer à 1,8 milliard le nombre de celles confrontées à une pénurie d'eau ». "Pour les $40 \%$ les plus pauvres, soit environ 2,6 milliards d'individus, nous nous trouvons à la veille de changements climatiques qui remettront en cause les perspectives de développement humain. "

81 S'agissant des services, les deux question stratégiques, dans une perspective de développement humain équitable et durable, sont celle de la prise en compte de leur écologie complète (voir la section 1) et celle de l'accès universel à des services essentiels associés à des droits déjà reconnus ou à conquérir. Mais aucune de ces deux questions ne peut être posée en isolant les services des autres activités et de leur organisation matérielle et spatiale. Par exemple, on n'aura pas de « services durables » dans des villes non durables, avec des transports ou des bâtiments non durables, et avec des prestataires dont les niveaux et modes de vie ne sont pas durables non plus.

L'exigence de durabilité porte une exigence d'égalité, qui concerne aussi les services. Mais cette double exigence sera source de conflits majeurs

L'exigence d'égalité va se poser en relation avec la notion de place (ou espace, ou empreinte) écologique disponible par personne. Les milieux aisés et les élites feront tout, au nom de leur utilité sociale supposée supérieure, pour conserver leurs places écologiquement dispendieuses en réduisant au maximum l'espace écologique utilisé par les personnes situées en bas de l'échelle. Mais elles seront toutefois en difficulté : les riches pouvaient utiliser l'argument, présent par exemple chez Adam Smith, et toujours réactualisé depuis par les élites, selon lequel la richesse des riches profite à tous en entretenant le dynamisme des affaires. Ce raisonnement reposait sur la vision d'une richesse économique matérielle extensible sans limites. Dès lors que la richesse contient une composante proprement vitale de ressources naturelles finies, l'argument ne tient plus: la richesse écologique des riches (reflétée par exemple par leur empreinte écologique) s'oppose frontalement au bien-être durable du plus grand nombre. La lutte des places pour l'accès à des ressources naturelles disponibles en voie de raréfaction se conjuguera à la lutte des classes pour le contrôle et la répartition de la production économique. On retrouvera la problématique de Fred Hirsch dans «Social limits to growth » (1977).

Les services seront directement concernés par ces revendications égalitaires dans la mesure où nombre d'entre eux sont non seulement associés à des droits universels (santé, éducation, mobilité, justice, logement, énergie, etc.), mais sont aussi, parfois, dépendants de ressources naturelles vitales (l'eau, l'énergie, l'espace...). La question sera donc posée de la tendance spontanée des marchés concurrentiels à produire ou reproduire des inégalités, de l'exclusion et des dommages écologiques. La "marchandisation concurrentielle » des services, propulsée notamment par l'AGCS (Accord Général sur le Commerce des Services) sera questionnée. Bien que l'époque soit à la «dérégulation » des 
services, la crise écologique pourrait bien exiger leur re-régulation et de fortes limitations de la logique concurrentielle actuelle à court terme qui conduit au dumping social et environnemental. Des biens communs environnementaux, ou «services de la nature ", devront également acquérir en droit un statut les préservant de la logique marchande lucrative. Ce statut sera le lieu d'enjeux sociaux majeurs car la mise hors marché concurrentiel ne suffit pas à garantir l'équité ni la durabilité écologique.

L'économie écologique en général et celle des services en particulier exigeront dans les décennies à venir une plus forte intervention publique et des financements publics plus importants, notamment dans les services. Pour trois raisons : 1) celles qui viennent d'être évoquées : la réduction des inégalités d'accès aux services de la nature et aux services fondamentaux de la solidarité et $\mathrm{du}$ bien-être, 2) pour lancer de nouveaux investissements qui seront à la transition vers l'économie écologique ce que les investissements dans les infrastructures et grands services publics de réseau ont été à l'économie de la révolution industrielle aux Trente Glorieuses, et, 3) parce que les comportements des consommateurs et des entreprises devront être réorientés en utilisant, parmi d'autres outils, des incitations, normes et taxations bien plus fortes, du local au global. Cela posera une autre question sociale qui est celle de savoir qui supportera, dans le monde et dans chaque pays, les efforts de reconversion, sur quelle base et avec quel taux d'effort. Il serait paradoxal que les pays, les catégories sociales et les peuples qui ont le moins contribué à nous faire franchir les seuils de non durabilité, et qui sont pourtant ceux qui en font déjà le plus les frais, ne voient pas reconnaître la dette écologique que leur doivent les "riches ", dont la richesse s'est construite en grande partie en surexploitant ou en pillant, entre autres, les ressources naturelles du Sud.

\section{NOTES}

1. ZenithOptimedia : http://www.lesechos.fr/info/comm/4657216.htm

2. http://www.citepa.org/emissions/nationale/Ges/Emissions_FRmt_GES.pdf

3. L'empreinte écologique d'une population est la surface de la planète, mesurée en hectares, dont cette population dépend, compte tenu de son mode de vie et des techniques actuelles, pour ses besoins en produits du sol (agriculture, sylviculture) et en zones de pêche, en terrains bâtis ou aménagés (routes et infrastructures), et en forêts capables de recycler les émissions de $\mathrm{CO}_{2}$.

4. Cette section doit beaucoup à des échanges avec Fabrice Flipo et Jean-Marc Jancovici, qui m'ont adressé remarques, suggestions, données et documents de référence sur des questions que j'ai découvertes récemment alors qu'ils les explorent depuis des années.

5. Greenpeace et site «planète urgence » : http://www.infosdelaplanete.org/1900/ combien-de-petrole.html

6. voir : http://www.pcinpact.com/actu/news/Un_m_deau_pour_1Go_de_RAM_.htm, citant des chercheurs de l'université des Nations Unies à Tokyo : http://

search.japantimes.co.jp/member/member.html?fe20030123sh.htm 
7. cf. Arnulf GRÜBLER, the Rise and Fall of Infrastructures, 1990, repris dans GIEC, 2001

8. Voir : http://www.lepapier.fr/cestlavie.htm

9. Elles concernent notamment les secteurs des spectacles vivants, de l'éducation et de la santé.

10. Avec une nuance : certaines productions agricoles ne sont pas appelées à suivre cette tendance moyenne. Par exemple, pour des raisons qui tiennent à sa forte empreinte écologique, l'élevage des bovins devrait connaître à terme une forte décroissance car il s'accompagne d'un haut niveau d'émissions de gaz à effet de serre.

11. http://www.jean-fourastie.org/temoin1.htm

12. Objection : le remplacement progressif de l'agriculture intensive par une agriculture durable, plus riche en emplois, orientée vers des marchés plus proches, n'est-il pas contraire à l'impératif de nourrir une population mondiale croissante ? Non, au contraire. D'abord, nous n'avons pas vraiment le choix, car sans de telles mesures, la population mondiale souffrira de catastrophes liées au dérèglement de l'environnement, et les plus pauvres seront aux premières lignes. Ensuite, la FAO elle-même affirme le rôle essentiel d'une agriculture durable pour nourrir l'humanité et maintenir des emplois « au pays » (http:// www.fao.org/newsroom/fr/news/2007/1000550/index.html), ce que confirme le beau livre de Bruno Parmentier (Nourrir l'humanité, La découverte, 2007), qui défend la souveraineté alimentaire comme un droit pour chaque peuple, contre le productivisme mondialisé.

13. http://www.negawatt.org/

14. Au sens de leur poids dans l'emploi, mais aussi au sens de leur diffusion sur tous les territoires et de leur accessibilité à tous.

15. D'après un récent rapport de l'OMI (Organisation maritime internationale), commenté par The Guardian, le transport maritime émettrait 1,12 milliards de tonnes de CO2 par an, soit $4,5 \%$ de l'émission anthropique globale, chiffre qui pourrait croître encore de $20 \%$ d'ici 2020. Ce chiffre est presque le triple de l'estimation retenue jusqu'à présent par le GIEC. Il approche du double des émissions du transport aérien. L'évaluation des autres polluants confiés à l'atmosphère par les navires (soufre, etc.) est plus fortement croissante encore : le fuel lourd généralement utilisé est un des pires polluants combustibles existant. Or le protocole de Kyoto ne tient pas compte du transport maritime. Voir : http:// www.guardian.co.uk/environment/2008/feb/13/climatechange.pollution

16. http://reports.eea.europa.eu/eea_report_2008_1/en

17. http://www.millenniumassessment.org/en/index.aspx

\section{RÉSUMÉS}

Pour construire une économie écologique des services, il faut d'abord s'intéresser aux bilans écologiques complets de ces activités. Il apparaît alors que l'immatérialité parfois supposée des services est un mythe. Il pourrait bien en résulter des freins à l'expansion future des services dans l'emploi, dès lors que leurs externalités environnementales seraient internalisées et que 
l'agriculture et l'industrie seraient réorientées sur une voie non productiviste. Mais ces bilans et prospectives doivent être effectués à un niveau sectoriel plus fin que la trilogie traditionnelle.

In order to build ecological economics of services, we need first to investigate the question of the environmental pressure that services exert and how to measure it. It appears that the alleged immateriality of services in largely a myth. Consequently, the trend towards increased servicesector employment could possibly be affected or even reversed, as far as environmental externalities are internalized and agriculture and manufacturing reoriented on a nonproductiviste way. But such an analysis ought to be carried out at a more detailed industry level than the traditional division in three sectors.

\section{INDEX}

Mots-clés : services, économie écologique, bilan écologique, loi de Baumol, productivité, emploi tertiaire

Keywords : ecological economics, environmental pressure, Baumol's law, productivity, service employment

\section{AUTEUR}

\section{JEAN GADREY}

Jean Gadrey est Professeur émérite à l'Université de Lille 1. 